\title{
Upstream waves and field line resonances: simultaneous presence and alternation in $\mathrm{Pc} 3$ pulsation events
}

\author{
J. Verõ ${ }^{1}$, H. Lühr ${ }^{2}$, M. Vellante ${ }^{3}$, I. Best ${ }^{4}$, J. Střeštik ${ }^{5}$, J. Cz. Miletits ${ }^{1}$, L. Holló ${ }^{1}$, J. Szendrõi ${ }^{1}$, B. Zieger ${ }^{1}$ \\ ${ }^{1}$ Geodetic and Geophysical Research Institute of the Hungarian Academy of Sciences, H-9401 Sopron, Pf. 5, Hungary \\ ${ }^{2}$ GeoForschungsZentrum Potsdam, Champ Project Office, Telegrafenberg, D-14473 Potsdam,. Germany \\ ${ }^{3}$ Dipartimento di Fisica, Universita dell'Aquila, Via Vetoio, I-67010 Copito-L'Aquila, Italy \\ ${ }^{4}$ GeoForschungsZentrum Potsdam, Adolf Schmidt Geomagnetisches Observatorium Niemegk, D-14823, Germany \\ ${ }^{5}$ Geofysikalni Ustav, AV ČR, Praha 4, Spořilov. Bočni II 1401, CZ-14131, Czech Republic
}

Received: 3 September 1996 / Revised 27 June 1997 / Accepted: 27 July 1997

\begin{abstract}
Based on a detailed study of Pc3 events at an array between $L=1.5$ and 3 in Central Europe, the authors found quick changes between upstream waves (UW, i.e. pulsation directly driven by UW) and field line resonance (FLR, i.e. azimuthal oscillations of geomagnetic field lines). The alternation of the two types is especially characteristic (and the UW part stronger) if the interplanetary magnetic field (IMF) is highly variable. Events due to field line resonance may have a structure consisting of multiple lines with frequencies differing by about $10 \%$, corresponding to neighbouring shells of field lines separated by about $100 \mathrm{~km}$ at the surface. This coincides with previous findings (about $10 \%$ at a meridional distance of $80 \mathrm{~km}$ ). The frequency of the UW type is well correlated with the frequency of waves in the interplanetary medium. Additionally, there are signals of unidentified origin which also seem to be influenced by IMF.
\end{abstract}

Key words Magnetosphere Physics - MHD waves and instabilities $\cdot$ Plasmasphere $\cdot$ Solar wind/magnetosphere interactions

\section{Introduction}

In the first six months of 1991, the geomagnetic observatories Niemegk (NGK), Nagycenk (NCK) and L'Aquila (AQU), lying approximately along a meridian ( $L$-values of the three stations at a height of $110 \mathrm{~km}$ are $2.25,1.87$ and 1.55 , respectively), carried out a pulsation campaign. Pulsations were recorded simultaneously between 0800 and 0900 UT, and then between 1400

Correspondence to: J. Verõ e-mail: vero@ggki.hu and 1500 UT. The campaign aimed at a detailed study of the two most important sources of Pc3 (10-45 s) and Pc4 (45-150 s), namely of waves driven directly by upstream waves (UW) originating in the fore-shock interplanetary space (Gul'elmi, 1974; Verõ, 1980, 1986, Russel and Hoppe 1981; Yumoto,1986, Střeštik, 1987, Yedidia et al., 1991, Villante et al., 1992, Verõ et al., 1994) and of waves due to field line resonance (FLR). FLRs (Southwood, 1974; Chen and Hasegawa, 1974) are generally characterized by nearly sinusoidal waveforms. The frequency (period) of pulsations with FLR origin is approximately constant at a station, independently of interplanetary conditions. There is a change in period, however, with magnetic latitude. When talking about FLR we think of azimuthal oscillations of the magnetic $L$-shells. Individual $L$-shells can oscillate quite independently at their eigenmodes. Due to the $90^{\circ}$ rotation in the ionosphere (Hughes, 1974), toroidal field line oscillations give rise to ground pulsations primarily confined to the geomagnetic north/south, H, component. We shall call pulsations with periods depending on geomagnetic latitude or $L$-value field line resonance or simply FLR in the following. The other type of pulsations, due to upstream waves, exhibits a less regular form. The spectra show broader peaks; the periods do not change with latitude but follow the interplanetary magnetic field (IMF) magnitude in average approximately according to the relation $T=170 / B$, where $T$ is the period (in s), and $B$ the scalar magnitude of the IMF (in nT). Therefore, these pulsations may be regarded as directly driven by UW. (It is to be remarked that pulsations from other sources may also have periods which do not depend on $L$-value and are not correlated with the IMF. One should, however, be cautious in the formal distinction of the two types, as both may come from the same primary origin.

In the following, the term impulse or $U W$ impulse is often used. Pulsation activity is mostly of variable amplitude, quite often wave packets follow each other rather regularly. These wave packets can have at least two different origins: they can be due to interference of 
signals with slightly differring frequencies, and due to realistically existing impulsive excitation (see Verõ and Cz. Miletits, 1994). In the following, an impulse is called a $U W$-impulse, if the period of the impulse does not change with $L$-value (or with latitude). It will be shown that the occurrence frequency of such UW-impulses increases with increasing variability of IMF, and thus they may originate from the interplanetary medium, while beats are artefacts of the interference without any special source.

In a first study of the collected material (Verõ et al. 1995), the average hourly spectra obtained at each of the three stations were studied from the point of view of distinguishing the two types of pulsations and to find characteristic parameters for them. It was confirmed that both sources of the pulsations have to coexist if we try to explain their observed characteristics, as had been found based on data of a former array, including two stations of the present array (Cz. Miletits et al., 1990). Sometimes FLRs had been identified only in a part of the chain of the three observatories, most often at $L$ values $2.5-3$. From the data of the same array it was supposed that the beating structure of the pulsations is due to interference of signals from neighbouring resonant shells. From the number of individual cycles within the beats, the difference in the eigenperiod of such shells was estimated to be about $10 \%$.

In the present paper we shall discuss in detail two selected intervals from the records of this array which were supplemented by records from the observatories Budkov (BDV, $L=2.0$ ) and Warnkenhagen (WRH, $L=2.5$ ). The first is situated between NCK and NGK, the second extends the array towards higher latitudes, but is still at mid-latitude. Moreover, records from Kakioka (KAK, $L=1.2$ ) were also used to see the effects of European pulsation bursts in the East-Asian sector, at a lower latitude. Due to the difference in LT, 0800-0900 UT is early evening at KAK, and no strong FLR activity is expected there. Sampling rate was at the different stations between 1 and $3 \mathrm{~s}$, and thus the lower limit of the dynamic spectra (10 s) is well above the Nyqvist period (2-6 s).

Based on the type of the pulsations seen at the different stations, 13 intervals were chosen for detailed investigation. The first selection was subjectively made so that the sample should include all distinguishable pulsation types (period changing with $L$-value, constant period everywhere, intermittent activity, etc.). For these intervals high-resolution dynamic spectra were produced from the available records of all stations. The "normal" dynamic spectra used in this study are computed with digital filters having centre period/ frequency steps of $10 \%$ and covering the whole range of the periods studied. The advantage of computing dynamic spectra with convolution filters is that the parameters can be more easily changed, adapted to the period range studied for example by changing the truncated length of the filters (see also the Appendix and further comments to come). The reliability of the power and dynamic spectra was checked by comparing spectra computed by different methods. From these intervals, two with variable spectra were selected for a detailed study of the quick changes in the pulsation regime. The 0800-0900 UT interval, 18 June was most thoroughly investigated, being of special interest from the point of view of quick changes in the occurrence of different pulsation types along the meridian. The results were then compared to simultaneous interplanetary data (IMP-8) concerning IMF magnitude and upstream wave activity. The 0800-0900 UT, 22 May event is also presented in some detail, as an event with several similar, but also some different characteristics.

\section{June event}

\subsection{Detailed study of the dynamic spectra}

In the following, records and dynamic spectra of the 0800-0900 UT, 18 June event are presented as an interval which contained simultaneously both types of Pc3-4 pulsations. Records of all the six stations were available, at WRH with a short interruption in the middle of the interval (0830-0835 UT). Due to some outstanding features, this interval is also called an event.

Amplitude spectra of the full 60 min show a continuous increase in the dominant period with $L$-value (Fig. 1). (The FLR periods were in the time of this study at the stations of the array: AQU, $15 \mathrm{~s}, \mathrm{NCK}, 22 \mathrm{~s}$, BDV, $26 \mathrm{~s}$, NGK, $32 \mathrm{~s}$, WRH, $41 \mathrm{~s}$. These values are based on a preliminary statistical estimation of the dominant period at each station.) At AQU, there is a 16s FLR peak, with a flat secondary maximum at about 32 s. At NCK, a rather broad maximum is at $23 \mathrm{~s}$ (FLR), this maximum is flat, especially towards longer periods, extending nearly to $30 \mathrm{~s}$. At BDV, the maximum lies at $28 \mathrm{~s}$ (FLR), and it is rather sharp. At NGK, the spectrum is again flat, with maxima around $65-70 \mathrm{~s}$ and at $34 \mathrm{~s}$ (FLR); at even shorter periods, there are no clear peaks, but the amplitudes are at a constant level down to $12 \mathrm{~s}$ - this part may include the second harmonic of the FLR. At WRH, the maximum is at $42 \mathrm{~s}$ (FLR), with an additional secondary peak at $22 \mathrm{~s}$ (second harmonic of FLR?) and a third small one at $13 \mathrm{~s}$. At KAK, there is a flat peak between 40 and $55 \mathrm{~s}$, with a secondary one at $32 \mathrm{~s}$, without any indication of FLR. There was in general no identifiable noise at any of the stations. Exceptions are small short-period impulses at NGK that may be caused by the minute time-marks. These impulses might add to the 65-70-s peak. The small 13-s peak at WRH may also be influenced by noise. It is to be remarked with regard to these characteristic periods that we did not taken into account each instrument's transfer function (which is everywhere near to flat in the period range studied), as we have limited our attention to the determination of dominant periods without comparing signal strength along the array. The inclusion of the instrument transfer functions would not change the position of the spectral peaks; it could, however, change the shape of the spectrum. Therefore, when discussing shapes of spectra, this fact should be remembered. 

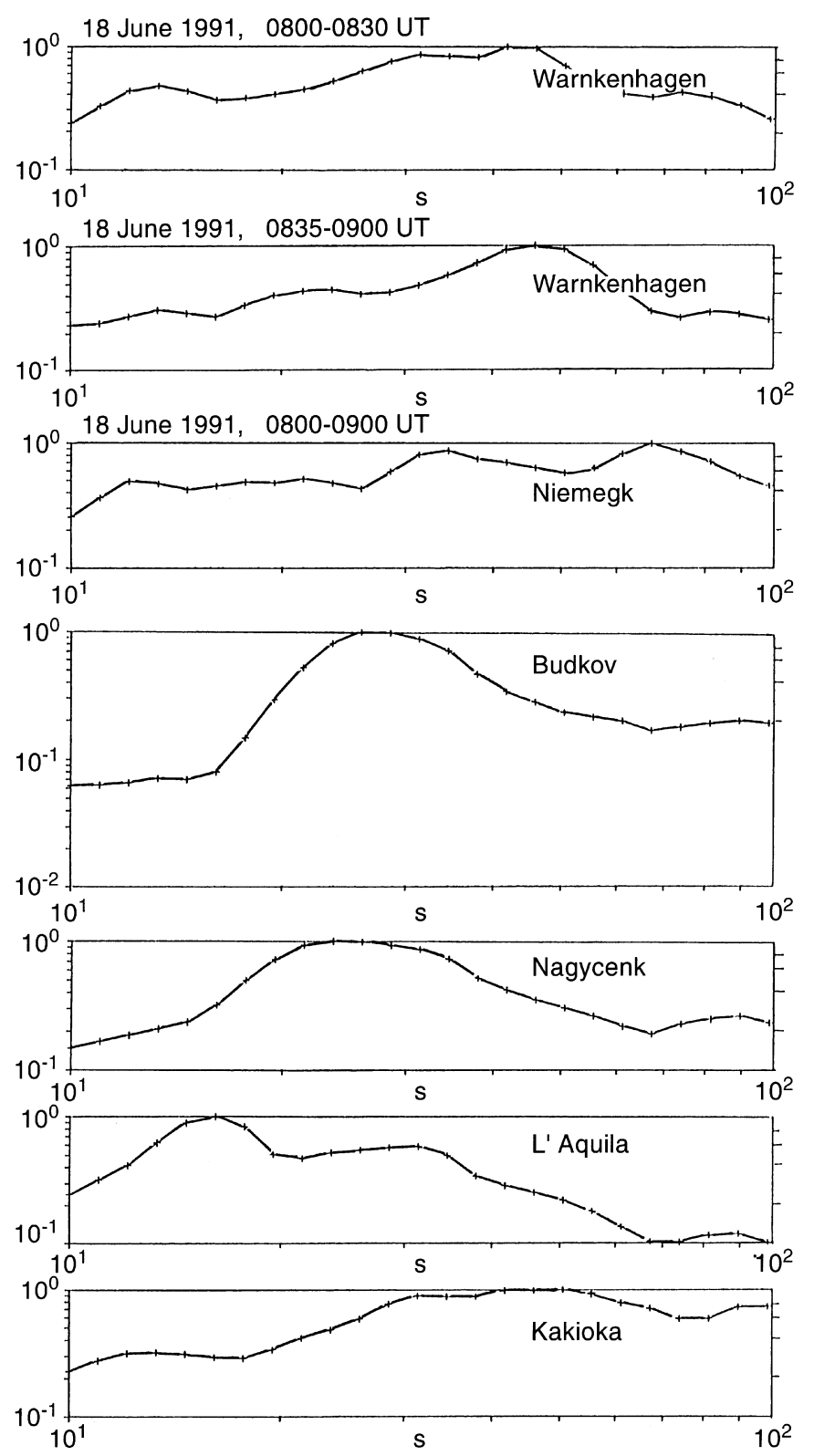

Fig. 1. Amplitude spectra of the 0800-0900 UT, 18 June, 1991, event at different stations. On the vertical axis, amplitudes reduced to a maximum amplitude 1 within the plotted range, on the horizontal axis periods in $\mathrm{s}$

Based on the hourly spectra, this event seemed to represent a clear example of pure FLR, perhaps with the addition of some activity with periods of about $30 \mathrm{~s}$ at several stations (supposedly UW due to the constant period at different latitudes). Dynamic spectra (Figs. 2 and 3) enable us to identify more details of the active period ranges.

AQU: two active period ranges: one at 13-18 s (continuous with a beating structure) and another one around $30 \mathrm{~s}$ (strong beats or impulses);

NCK: continuous activity around $23 \mathrm{~s}$, sometimes $(0830,0852)$ with a second active range at about $28 \mathrm{~s}$;

BDV: continuous activity at about $28 \mathrm{~s}$ with beats;
NGK: nearly continuous 60-70 s, rather continuous $\sim 30$ s and some 12-20 s activity, with "bridges" between them;

WRH: strong activity around $45 \mathrm{~s}$, in particular after 0835 ;

[KAK: slight activity of pulsations with period increasing $(25-40 \mathrm{~s})$ in time].

Thus, dynamic spectra indicate also some 30-s activity, which, as it is at least sometimes present at most stations, might be connected with upstream waves. Afterwards we could identify the greatest number of UW impulses during this event among all studied. Nevertheless, the dominant bands correspond to FLR as expected, and they mostly have strong beating structure (spots). Amplitude and phase diagrams of the (filtered) series confirm that the spots can result from beating, i.e. from the interference of two neighbouring frequencies, as at moments of amplitude minima the phase skips by $\pi$ (see Fig. 4 for the 25.9-s series of BDV). If the dynamic spectra from the various stations are overlayed, some of the spots of high activity rather surprisingly cover each other at several stations, or even at all of them. In other cases the spots appear shifted according to the expected change of the FLR period with latitude. To be able to study these spots of high activity, seven time-intervals were selected when Pc3-4 activity peaked everywhere along the array. In each of them, either spectra of short intervals (some minutes long) were computed, or instantaneous amplitudes were taken from the dynamic spectra, when the length of the interval is determined by the length of the filter (actually about $1 \mathrm{~min}$ ). The following Table 1 gives the results of this detailed study.

[Note that the 12- and 60-s periods at NGK may be partly due to the minute time-marks, and they are therefore not fully reliable, but a 60-s signal may also be trapped plasmasphere mode (Samson et al., 1992).]

The spectra of the selected short intervals indicate a predominance of the UW-type before 0820; then up until 0839, FLR gets stronger and stronger, at which time UW dies away, and reappears immediately afterwards; towards the end UW again gets weaker, with continuously strong FLR. Concerning UW, the periods observed at the array indicate a rather strong change of the period from 22 to $42 \mathrm{~s}$.

As regards the distant Asian station KAK, whenever events of UW pulsations were present at the European stations, there was, though mostly rather weak, some indication in the KAK records; impulses of FLR resonance were absent there. This is especially clear in the case of the 0836-0838.5 interval, when FLR activity was present along the array, without any indication at KAK.

Two facts are remarkable during the 18 June event: at first, UW- and FLR-type pulsations change quickly from one type to the other, so spectra of consecutive intervals may differ very significantly. The most eminent examples are the events at 0814 (UW) and at 0820-0823 (FLR) or at 0836-0838.5 (FLR), and that at 0839-0842 (UW). Figure 5 shows latitudinal cross-sections through the 0814 and the 0820 to 0823 events representing the two extremal conditions found during this interval. 

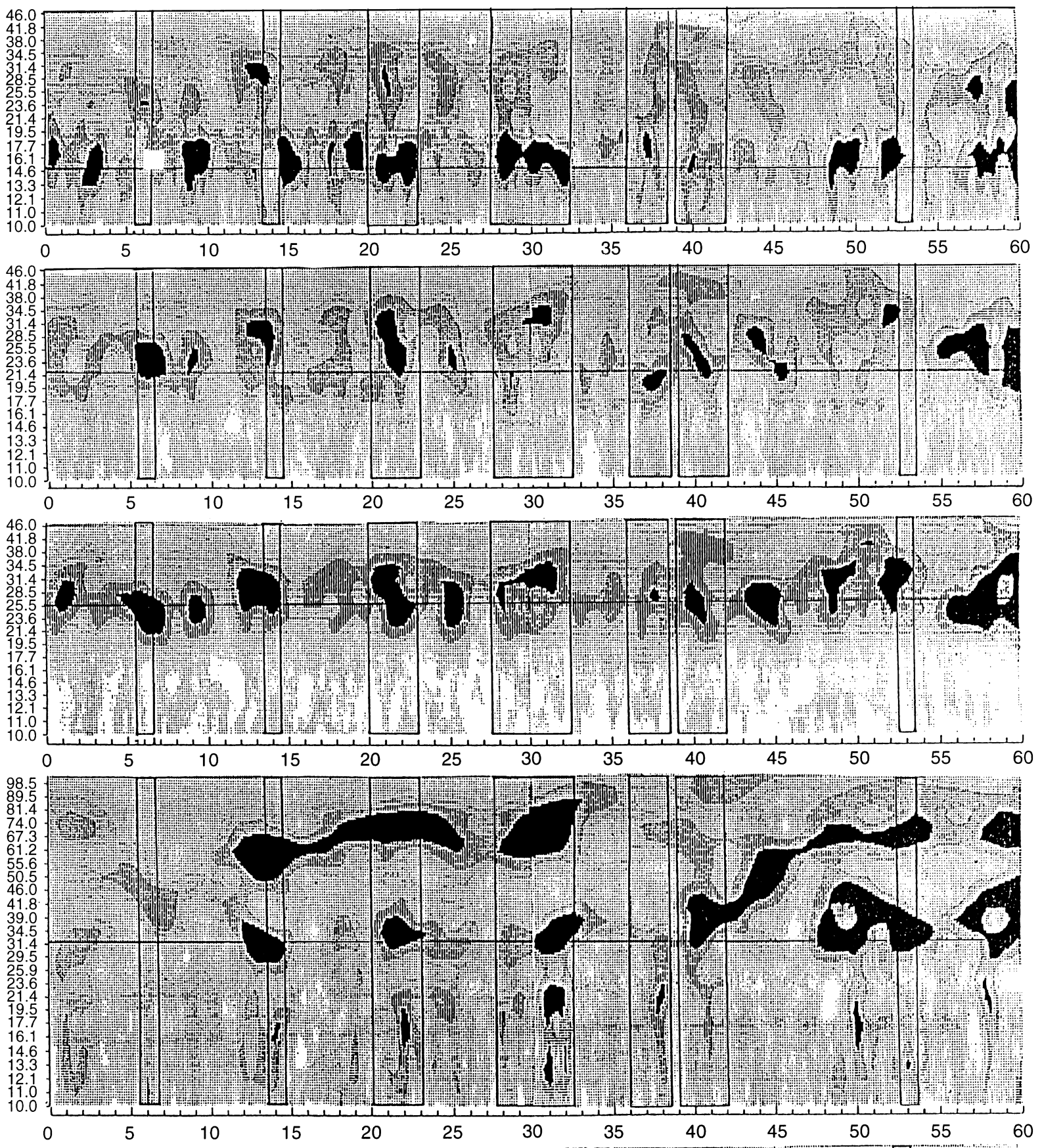

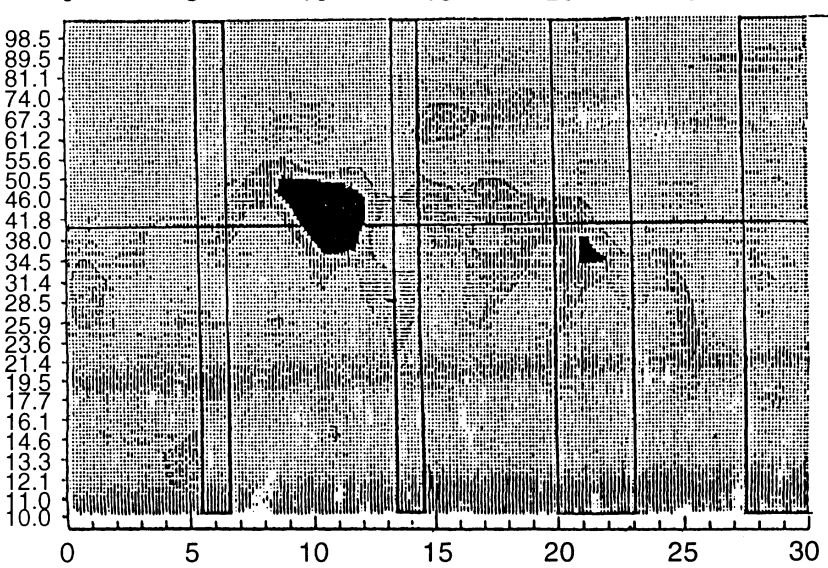

Fig. 2. Dynamic spectra of the 0800 to 0900 UT, 18 June, 1991, event (from top to bottom) at AQU, NCK, BDV, NGK, WRH. Different symbols (from black to white) correspond to steps in the amplitudes

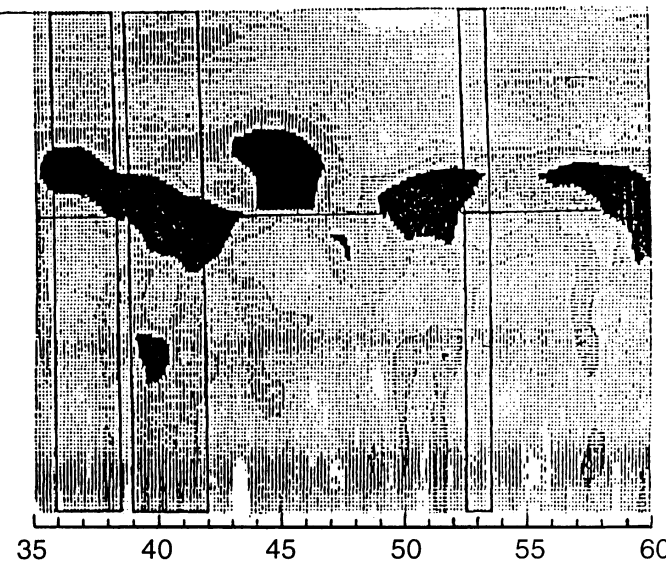

1.8 to 2 times at different stations. Vertical lines indicate intervals when amplitude spectra were determined, horizontal lines indicate the characteristic field line resonance period at the corresponding station 


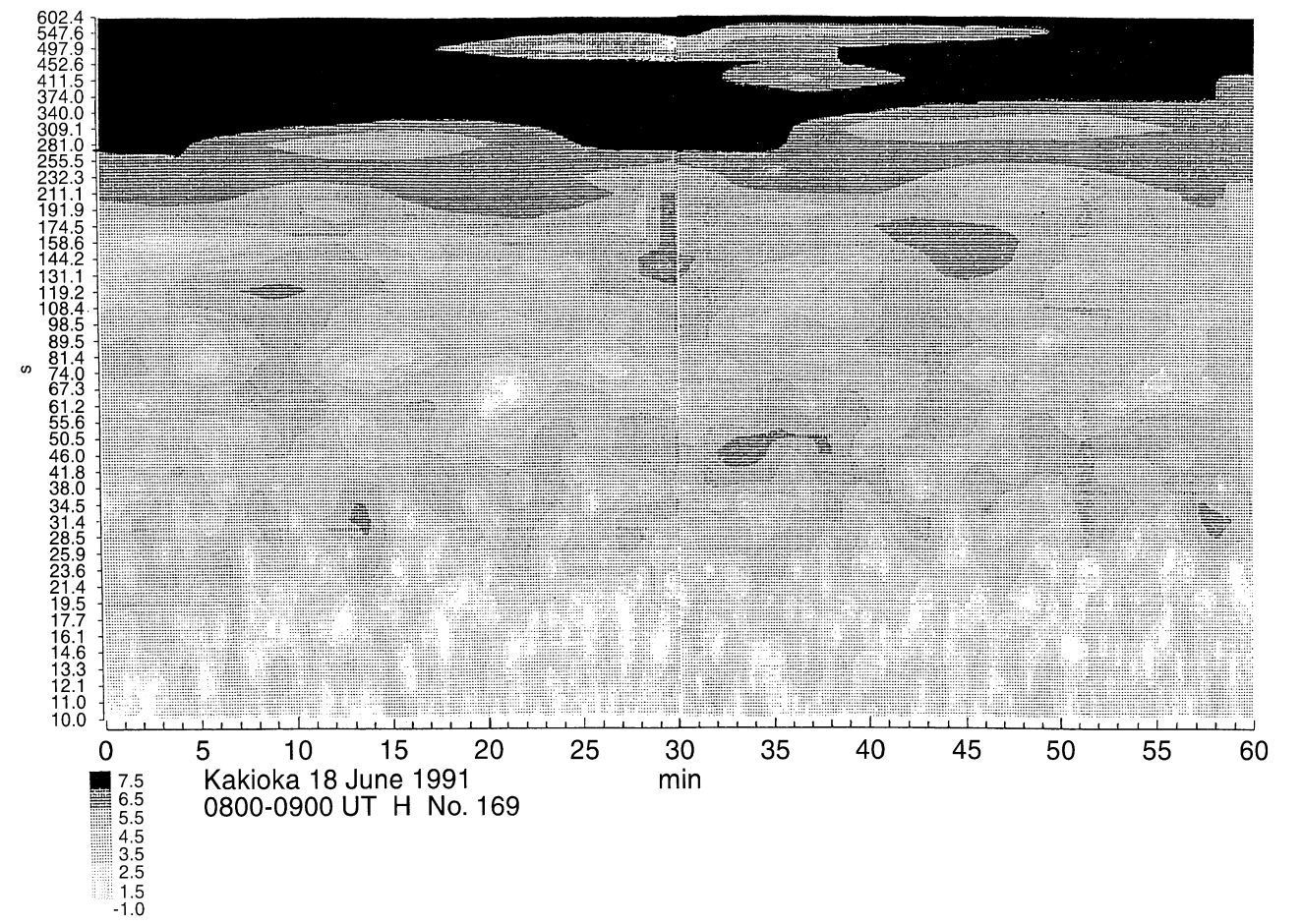

Fig. 3. Dynamic spectrum of the 0800 to 0900 UT, 18 June, 1991, event at KAK
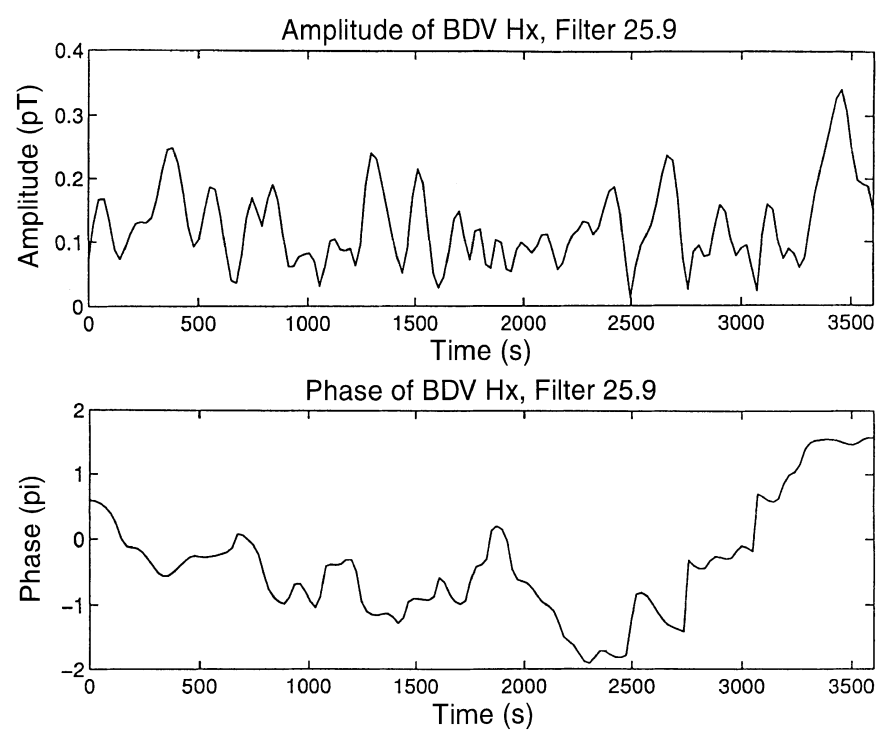

Fig. 4. Amplitude and phase of the 25.9-s filtered component at the station BDV with phase skips of $\pi$ at amplitude minima

During the first section, there was strong UW activity (28 s everywhere) with some trace of FLR only at NGK; during the second, there was an FLR main peak at each station. At NGK, the second harmonic was also present, with a somewhat smaller amplitude, and 40-s activity, possibly of UW origin, was observable at each station. The change between the two types is abrupt, taking place within, a few minutes or even less, and it clearly indicates the quickly changing excitation of UW and FLR pulsations.

The other remarkable fact is that originally the interval 0800-0900 UT, 18 June, was classified as belonging to the FLR type. The detailed study, however, revealed many UW impulses. The UW character does not appear dominant in the spectra of the full interval, since UW impulses have short duration and often change period.

As Fig. 6 shows, the period of the pulsations, tentatively called UW, changed with time. In general, they followed the changes of the IMF field strength with a shift of $5-10 \mathrm{~min}$ as observed by IMP-8, but the $B$ values actually observed are by a factor of about 1.5 higher than those estimated from the UW periods. IMP8 was at that time at a position (solar ecliptic coordinates) $X:-8$ to -9 Earth radii $\left(\mathrm{R}_{\mathrm{E}}\right), Y:+29.5$ to $+29.8 \mathrm{R}_{\mathrm{E}}, Z:+6$ to $+8 \mathrm{R}_{\mathrm{E}}$ and 5 -min averages of the magnetic field readings were used. A reasonable agreement between observed and predicted wave period is obtained if we use the relation $255 / B$ and allow for a propagation delay of 5 (or 10) $\mathrm{min}$. This is not identical to the simple formula $T=170 / B$, and makes the UW origin somewhat questionable, nevertheless, high-resolution (1.28-s) IMP-8 data confirm the close correlation between surface pulsation activity and interplanetary waves during this interval. Moreover, the spacecraft was outside the dusk flank of the magnetosphere on the nightside: however, this and some other events studied confirmed the possibility of an interplanetary medium surface correlation of the pulsations even in such cases.

The high-resolution IMP-8 data (Fig. 7) show clear Pc3 activity, especially in the $y$-and $z$-components. A shift to longer periods is also evident in the last $20 \mathrm{~min}$. Power spectra evaluated every $5 \mathrm{~min}$ with the maximum entropy method at order 15 (Fig. 8) for the $y$-component always show a clear peak in the Pc3 band.

Five-minute averages of the IMF intensity (top panel, time shifted backwards by $10 \mathrm{~min}$ ) and the central period of ground pulsations taken from Fig. 6, as well as 
Table 1. Characteristics of the pulsations in selected short intervals: bold face indicates dominant types, In the case of 0806,0814 and 0853 UT, instantaneous amplitudes were used, but due to the length of the filter, the sampled interval is in the critical period range about 1 min long, as indicated

\begin{tabular}{lll}
\hline UT & characteristics at stations & diagnosis of the spectra \\
\hline $0805.5-0806.5$ & KAK: max. 24 s, very small & UW: with periods 19-24 s, periods at NGK and WRH are \\
& AQU: max. 23-24 s, secondary max. 14 s & somewhat shorter \\
& NCK: max. 24 $\mathrm{s}$ & FLR: slight indication at AQU, stronger at NGK, but the \\
& BDV: max. 24-25 s & period is longer than normal, not separable at NCK and \\
& NGK: $\max .45 \mathrm{~s}$, secondary 12 s, small peak 22 s & BDV, next to nothing at WRH
\end{tabular}

$0813.5-0814.5$

RH:max.19 s, secondary 12 and (small) $42 \mathrm{~s}$

KAK: max. $32 \mathrm{~s}$, stronger

AQU: max. $30 \mathrm{~s}$, secondary max. $12 \mathrm{~s}$

NCK: max. $28 \mathrm{~s}$, small secondary $13 \mathrm{~s}$

BDV: max. 28 s NGK: three maxima, 16, 30 and $60 \mathrm{~s}$

WRH: low max. $30 \mathrm{~s}$, secondary at $48 \mathrm{~s}$

0820-0823

KAK: no max., some $30 \mathrm{~s}$ activity

AQU: max. $16 \mathrm{~s}$, flat secondary around $30 \mathrm{~s}$

NCK: flat max. at 23-32 s

BDV: $\max .28 \mathrm{~s}$

NGK: max. $34 \mathrm{~s}$, secondaries $70,18 \mathrm{~s}, 12 \mathrm{~s}$

WRH: max. $35 \mathrm{~s}$, flat at shorter periods

$0827.5-0832.5$

KAK: max. $50 \mathrm{~s}$, small secondary $30 \mathrm{~s}$

AQU: max. $16 \mathrm{~s}$, secondary $32 \mathrm{~s}$

NCK: max. $32 \mathrm{~s}$, secondary $23 \mathrm{~s}$

BDV: max. $32 \mathrm{~s}$, small secondary $23 \mathrm{~s}$

NGK: max. $65 \mathrm{~s}$, secondaries $32 \mathrm{~s}, 21,12 \mathrm{~s}$

WRH: no record (in the first part $32 \mathrm{~s}$ )

0836-0838.5

KAK: $\max .50 \mathrm{~s}$

AQU: flat max. $18 \mathrm{~s}$, small secondaries $22,34,45 \mathrm{~s}$

NCK: max. $20 \mathrm{~s}$, secondaries $32 \mathrm{~s}, 45 \mathrm{~s}$

BDV: max. $28 \mathrm{~s}$, small secondaries $22 \mathrm{~s}, 35 \mathrm{~s}$

NGK: max. $75 \mathrm{~s}$, secondaries $33 \mathrm{~s}, 19 \mathrm{~s}$

WRH:max. $50 \mathrm{~s}$, small secondary $25 \mathrm{~s}$

0839-0842

KAK: $\max$ at $38-40 \mathrm{~s}$

AQU: max. $15 \mathrm{~s}$, strong secondary $42 \mathrm{~s}$, also $23 \mathrm{~s}$

NCK: max. $23 \mathrm{~s}$, secondary $42 \mathrm{~s}$

BDV: max. $27 \mathrm{~s}$, strong secondary $42 \mathrm{~s}$

NGK: max. $42 \mathrm{~s}$, secondaries $23 \mathrm{~s}, 65 \mathrm{~s}$

WRH:max. $42 \mathrm{~s}$, strong secondary $22 \mathrm{~s}$

0852.5-0853.5 KAK: $\max .33 \mathrm{~s}$ and $45 \mathrm{~s}$

AQU: max. $16 \mathrm{~s}$, flat up to secondary max. $36 \mathrm{~s}$

NCK: max. $22 \mathrm{~s}$, secondaries $36 \mathrm{~s}$, (52 s)

BDV: max. $35 \mathrm{~s}$, flat towards shorter periods

NGK: max. $35 \mathrm{~s}$, secondaries $13 \mathrm{~s}, 70 \mathrm{~s}$, small $22 \mathrm{~s}$

WRH: max. $50 \mathrm{~s}$, secondary $35 \mathrm{~s}$
UW: with periods 28-30 s everywhere along the array FLR: not identifiable, the short period peak at NGK may be second harmonic (?), a trace at WRH (?)

UW: with periods $30-35 \mathrm{~s}$, longer at NGK and WRH FLR: clear at $\mathrm{AQU}$, present in a flat peak at $\mathrm{NCK}$, at $\mathrm{BDV}$, shift of the peak, at NGK coinciding with UW, at WRH, nothing

UW: 32 s, not too strong, but everywhere present FLR: strong at AQU, NCK, BDV, coinciding with UW at NGK

UW: slight trace at 32 s FLR: everywhere observed, but not very strong

UW: at 38-42 s, rather strong, especially at NGK and WRH

FLR: dominating in the southern part of the array, observable till NGK, coincides with UW at WRH

UW: $35 \mathrm{~s}$, weak indication at AQU and NCK, strong at BDV, at NGK coincides with FLR

FLR: strong everywhere, at BDV peak masked by the UW source spectrum upstream wave periods of the $y$-component from Fig. 8 are compared in Fig. 9. The correspondence is best for this shift, though 5-min shift backwards also gives acceptable coincidence. Thus the interpretation in terms of upstream waves of peaks identified in the ground spectra at latitudinally spread stations is reinforced by the direct comparison with the wave characteristics in the vicinity of the magnetopause, in the present case at the dusk flank of the magnetosphere.

The frequency of the main peak of the y-component is well correlated with the $B$ intensity: the correlation coefficient (for 11 points) is 0.74 and the regression analysis provides the equation: $T=237 / B$ where the constant of 237 is not far from the previously estimated value of 255 .
Concerning the amplitudes of the pulsations, the interplanetary activity has some features common with the ground activity, such as a switch-off in the middle of the interval, and a strong increase towards the end which is then followed by very high activity in the interplanetary medium around 0905 UT (no longer included in Fig. 1).

\subsection{Dynamic spectra of the event with very high frequency resolution}

Digital convolution filters used for the production of the dynamic spectra have characteristics which are to be explained before discussing this event further (see also 

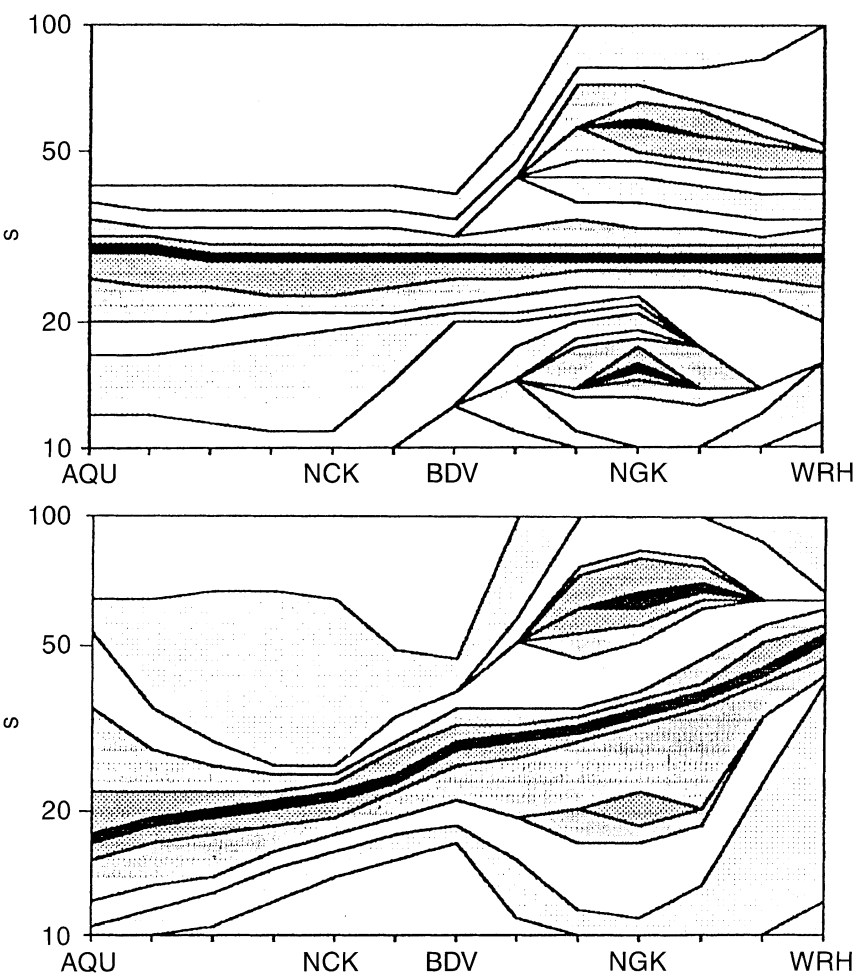

Fig. 5. Cross-sections from dynamic spectra through all stations at 0814 (top) and for the interval 0820-0823 (bottom). At each station, the spectra are normalized so that the maximum in the range 10-50 s is 1 , as in Fig. 1. Isolines are plotted at distances of 0.2 units

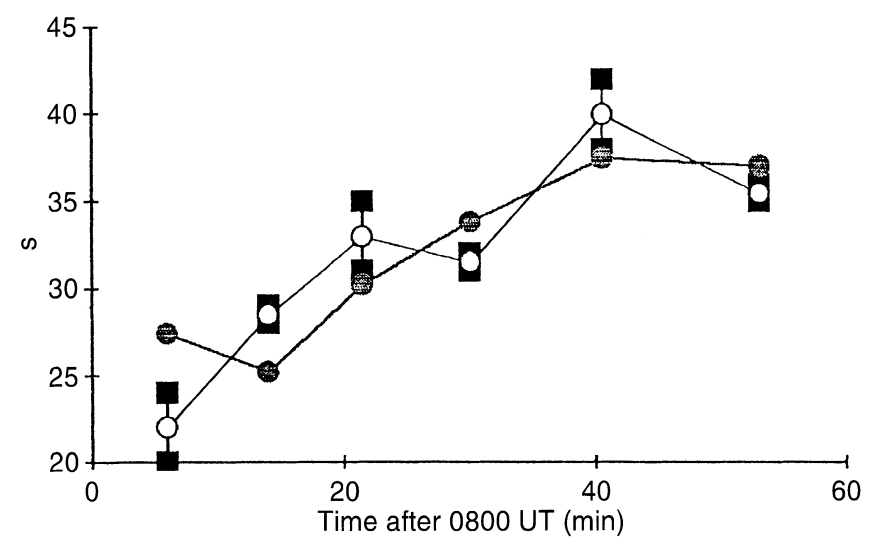

Fig. 6. The 0800 to 0900 UT, 18 June event: periods of pulsations supposedly originating from upstream waves. The range of periods observed along the array is indicated for each event when UW-type pulsations were identified. The IMF magnitude is represented (full circles, solid line) as $255 / B$, and shifted backward in time by 5 min

the Appendix). Theoretically, convolution filters have infinite length. For practical reasons, filters must be truncated. Truncation somewhat alters, however, the form of the transfer function. If the truncation is too strong, the frequency/period resolution deteriorates, but the time resolution improves (shorter filters are possible). In the case of less heavy truncation, the frequency resolution is better, but the time resolution gets poorer (longer filters). Thus, the actual filter used is always a trade-off between time and frequency resolution. For given time and frequency/period scales, the "best" filter is that one in which the spots in the dynamic spectrum are roughly circular at the given scales, meaning the same resolution in both directions. (Scales are always to be chosen based on practical experience. In the present case, the period width of the spots, be they impulses or beats, and their duration determine the scales, when a period difference of $10 \%$ corresponds to $1 \mathrm{~min}$, both being near to the average values of the corresponding parameters for such impulses or beats. Moreover, the applied $10 \%$ step in period is equal to the period difference of neighbouring resonant shells). For special purposes, e.g. for the exact determination of the moment of an impulse, one of the resolutions can be increased, but similarly to Heisenberg's relation, the resolution in the other direction gets poorer. In the case of the dynamic spectra presented in Figs. 2-3, the resolution is "circular" for periods around 20-30 s, i.e. for the most common periods of the UW-type pulsations and also for FLR around $L=2$. That means that if these filters are used, pulsation beats which may originate from the interference of neighbouring shell resonances (Verõ and $\mathrm{Cz}$ Miletits, 1994) exhibiting period differences of roughly $10 \%$, cannot be resolved. For this purpose, the frequency resolution must be increased at the cost of the time resolution. Figure 10 shows such dynamic spectra for the present event from NCK and NGK with increased frequency resolution. The maxima are elongated in the direction of the time-axis as expected. These features of the dynamic spectra do not yield a final argument either in favour of impulsive events, or for interference of neighbouring periods, as their form is simply a consequence of the mathematical treatment. If we have interference of two neighbouring waves, beats will result in case of high time resolution and individual bands in case of high frequency resolution (as seen in Fig. 10). Nevertheless, there are additional features in these dynamic spectra which are of interest in both cases, such as (1) the distance between the simultaneously active periods, and (2) moments of the changes of the interference structure. That is why these somewhat exceptional dynamic spectra are presented here. In the following we discuss in detail two features for the present event.

The very high resolution spectra reveal that the typical separation of the "lines" (not only during the selected events) is everywhere about $10 \%$, with a range between $7 \%$ and $14 \%$ (Fig. 11). (The distribution is bimodal, with two peaks at $10 \%$ and $13 \%$, but the minimum between them is not significant.

This value of the difference between lines, being around $10 \%$, is the same as deduced previously from the beat frequency of FLR (Verõ and Cz Miletits, 1994). Thus it is possible to interpret these "lines" as resonance frequencies of neighbouring shells (correspondingly, the estimated shell width at the surface is the same as previously found, namely about $100 \mathrm{~km}$ ). In such a case, however, it should be expected that lines found in the dynamic spectra of any two neighbouring stations coincide within an acceptable error limit. In the first part of the interval this is not the case, there are few 

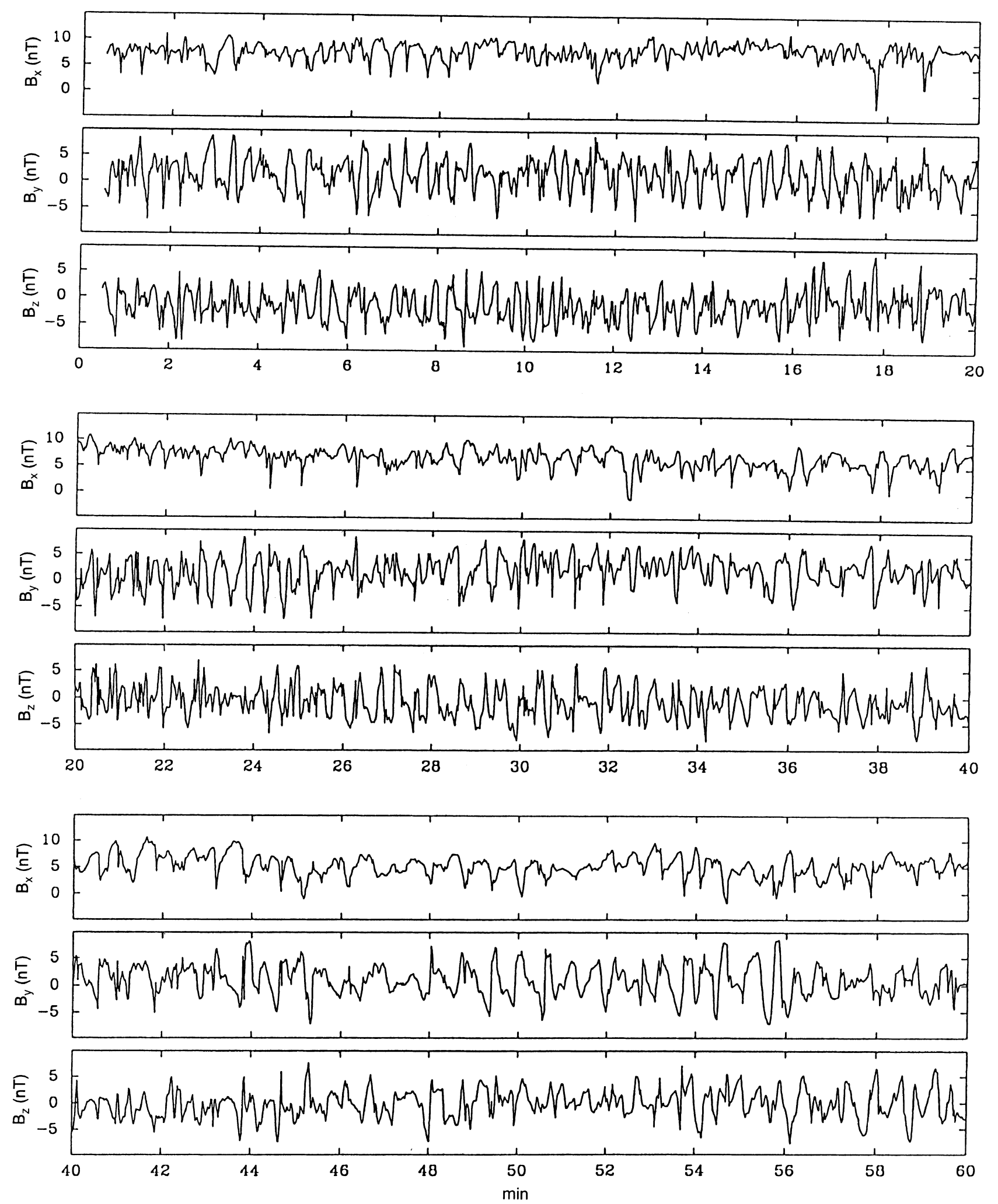

Fig. 7. High-resolution (1.28-s) IMP-8 data of the interplanetary magnetic field for the interval 0800-0900 UT, 18 June 1991

apparent coincidences. In the second part, when FLRs were more effective, there are several lines in the two spectra which coincide within an error limit of about $\pm 1 \mathrm{~s}$. The period of many other lines differs by about $10 \%$, as would occur if the two stations see only one (but not the same) of the two adjacent shells. Thus, the possibility of the direct detection of the shell structure of the FLR based on the pulsation records of a surface station at mid-latitudes should not be excluded in spite of several difficulties: the mathematical treatment does 
Table 2. Common spectral lines at station pairs

\begin{tabular}{lccccc}
\hline & AQU & NCK & BDV & NGK & WRH \\
\hline AQU & 16 & 4 & 5 & 3 & 0 \\
NCK & 4 & 18 & 10 & 8 & 4 \\
BDV & 5 & 10 & 17 & 3 & 0 \\
NGK & 3 & 8 & 3 & 28 & 6 \\
WRH & 0 & 4 & 0 & 6 & $(16)$ \\
\hline
\end{tabular}

not allow us to accept the picture obtained from the dynamic spectra with very high frequency resolution as a unique solution.

The number of lines which correspond with each other is given for each pair of stations in the following table. The counting is subjective and depends on the limits chosen for lines, nevertheless, the ratios are stable, as found by a few experiments. In a few cases, lines are present at more than two stations. The total number of counted lines at each station is also given (e.g. at the crossing point AQU-AQU).

A significant proportion of the lines, amounting to about $50 \%$, have a partner at some other station, especially at a neighbouring one, as e.g. at NCK and
BDV. At both ends of the array, i.e. at AQU and WRH, this amount is much less, about $20 \%-30 \%$.

The other interesting feature of these dynamic spectra is that in some cases the appearance of UW activity seems to terminate previous FLR structures and to excite new ones.

At NGK such changes are not very obvious, but even there they do appear; for example, the FLR lines change somewhat around 0830 and there are several new lines which start during the selected "short intervals" (e.g. a 60-s line at 0806, a 30-s line at 0820 and a 38-s line at 0837), afterwards they mostly soon die away.

At NCK abrupt changes are more prominent, especially at 0820 when a three-line structure turns to a twoline one; further, even if less spectacular, events are found at 0814, 0830 and 0853 . The appearance of new lines and their fading is also observable on several other occasions.

At AQU (not shown here), several impulses excited new lines and in some cases previously existing lines died away. Here, the 16-s FLR line is present from 0806 till 0833; both moments (start and end) coincide with a selected "short interval". Some appearances and disappearances of neighbouring lines (around the 16-s FLR)
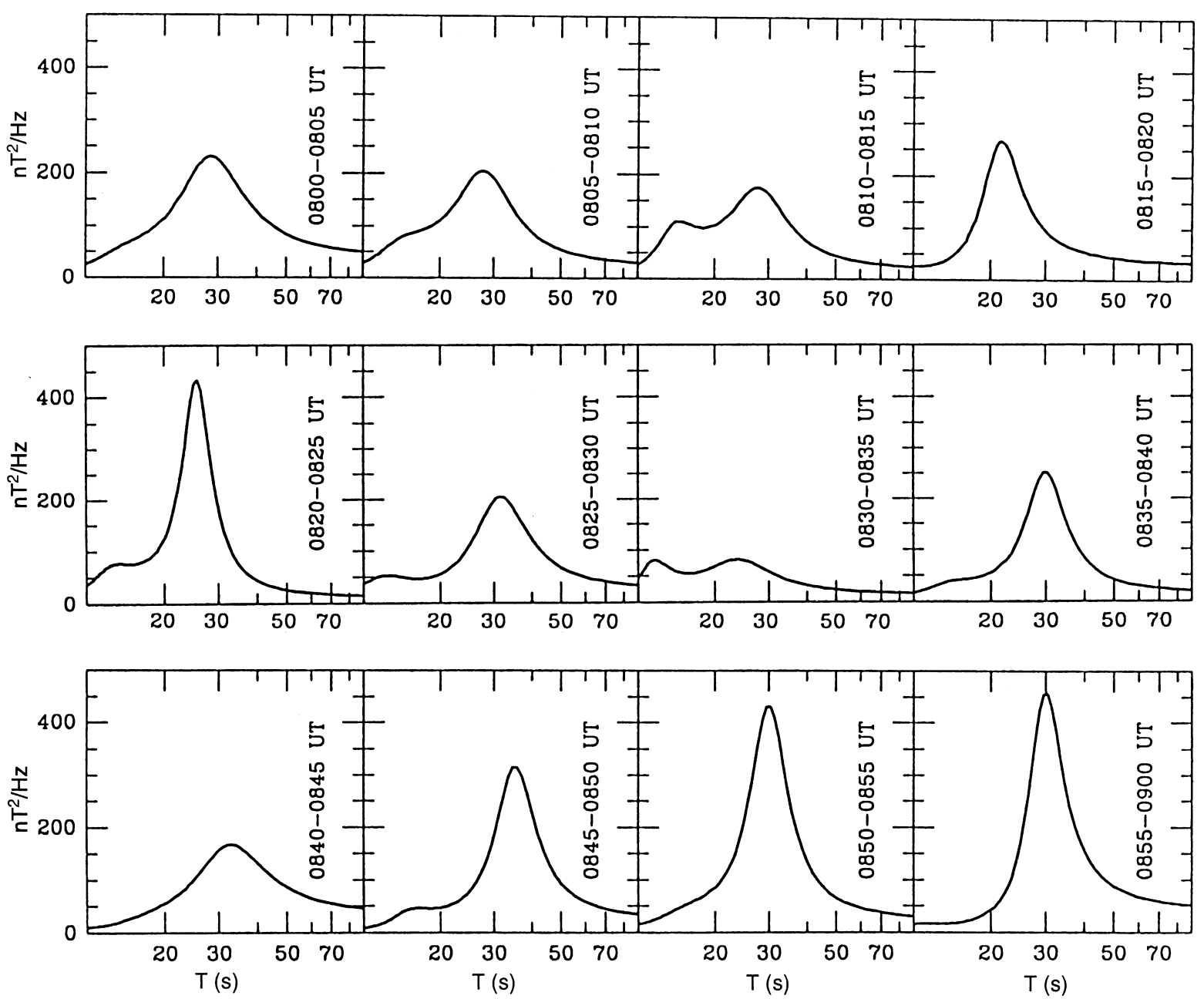

Fig. 8. Power spectra for the $y$-component of the IMF evaluated every 5 min, with the maximum entropy method at the order 15 

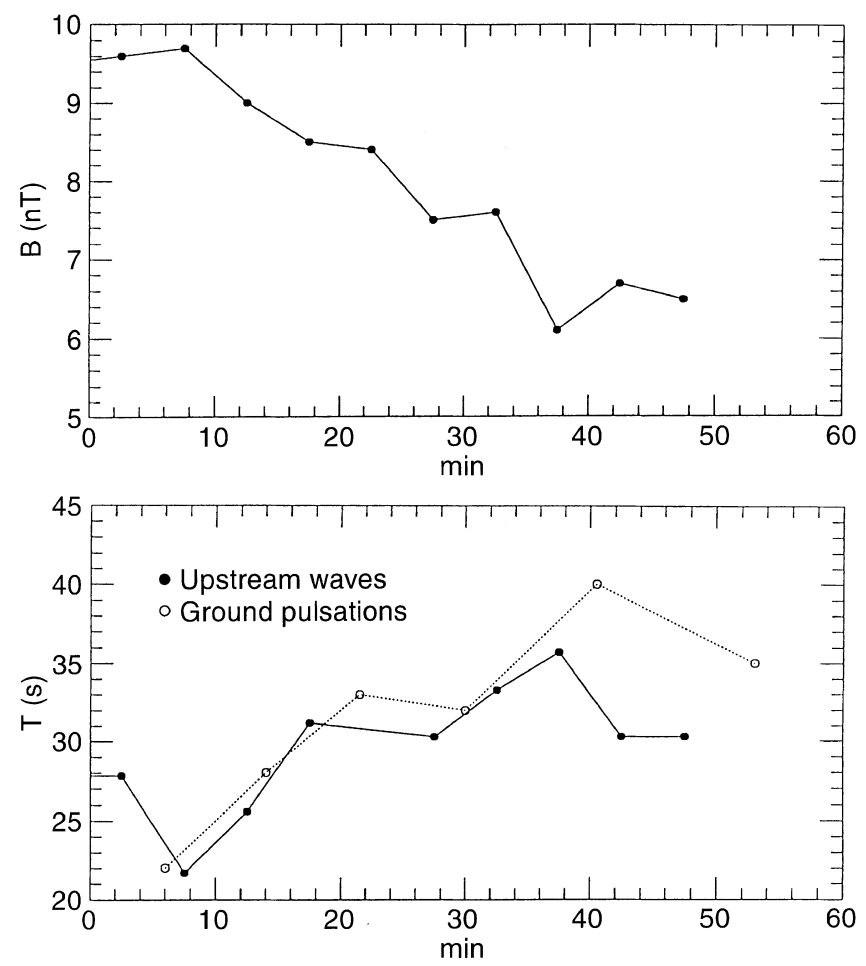

Fig. 9. Top panel: 5-min averages of the IMF intensity (time shifted backwards by $10 \mathrm{~min}$ ): bottom panel: central period of ground pulsations (empty circles, from Fig. 6) and upstream wave periods from Fig. 8 (full circles)

are connected to such events too. Thus, e.g. the 16-s line reappeared at 0846 and lasted till the end of the interval.

At WRH (not shown), the FLR line died away around 0820 , and only the UW signal remained. During the gap in the record, a very pronounced long-period structure developed, possibly due to the fact that the

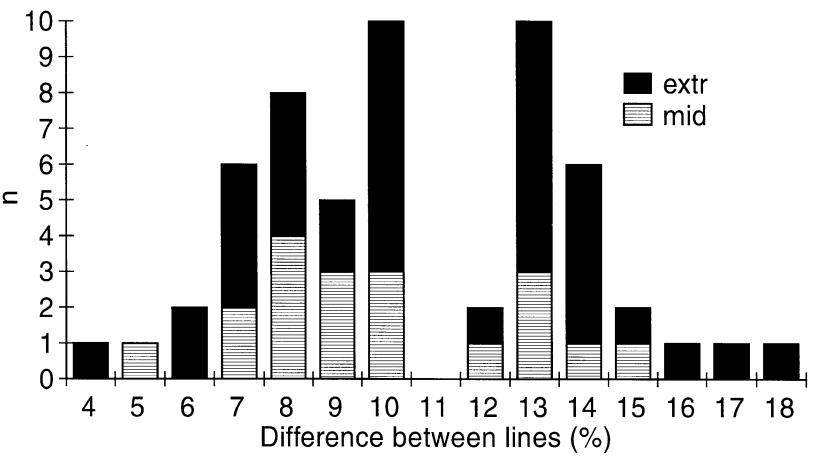

Fig. 11. Distribution of the distance between adjacent lines in the high frequency resolution dynamic spectra in percent (mid: NCK and $\mathrm{BDV}$, extr: all others)

supposed UW signals also had then long-period components, near the local FLR period at WRH. Around 0840, the periods at NGK and WRH indicate a very well developed FLR structure.

Thus transitions between different structures are connected to impulses, when previous structures are terminated and new ones come up. This fact leads to the supposition that both types have the same primary origin and resonance occurs at latitudes where the UW spectrum has enough power at the local resonant frequency.

From the study of a single event, i. e. 0800-0900 UT, 18 June 1991, which is exceptional due to the fact that the greatest number of individual UW impulses were found within $1 \mathrm{~h}$ from among all intervals studied, the following conclusions can be drawn:

1. Upstream wave events (i.e. events when the period does not change with $L$-value) are imbedded in intervals of strong pulsation activity, even if the
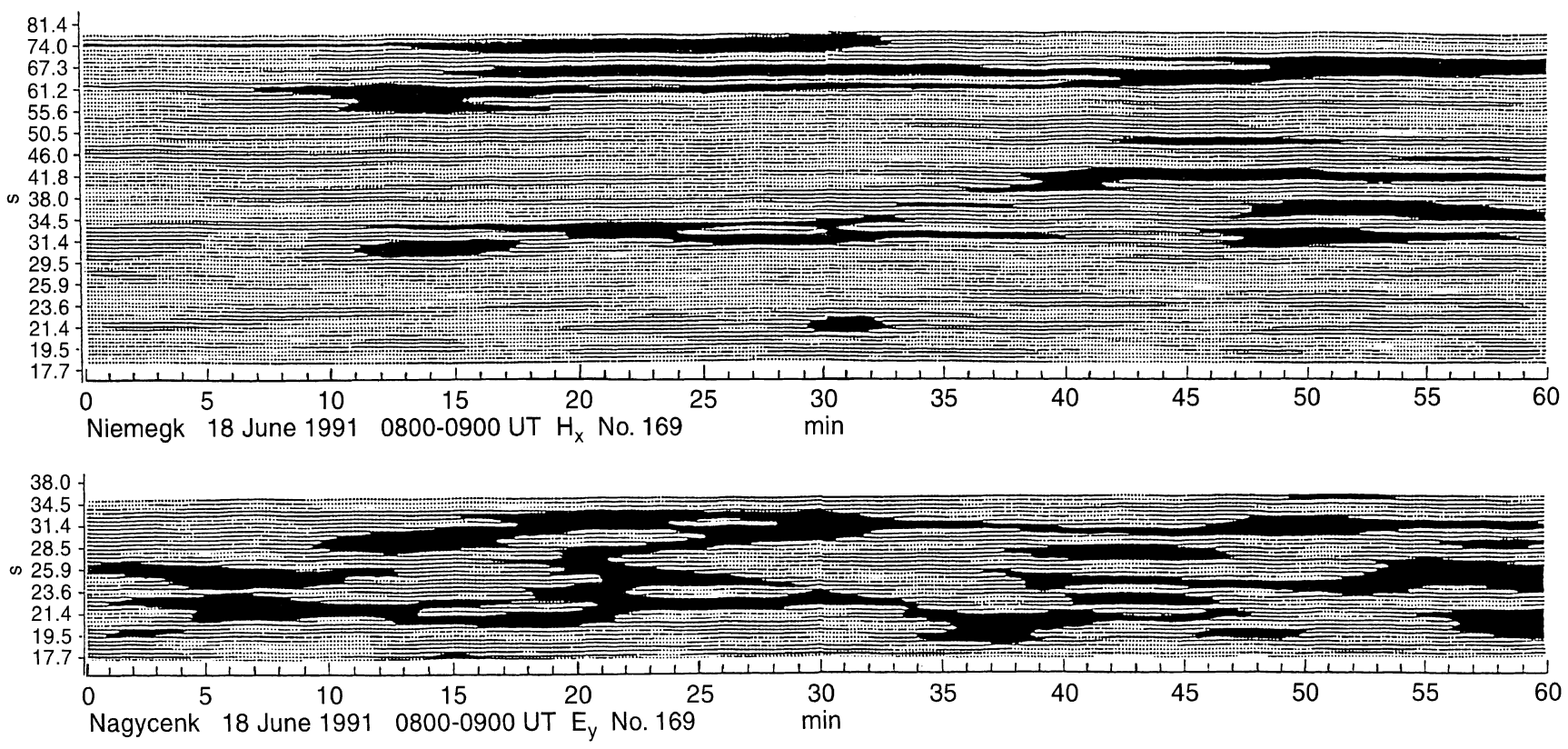

Fig. 10. Dynamic spectra of the 0800-0900 UT, 18 June 1991, event with increased frequency resolution at NCK and at NGK. Different symbols mean here amplitudes differring by a factor of 2 
activity is mostly of FLR origin. The two types, FLR and UW activity, may alternate quite quickly, i.e. within a few minutes, but they may also appear simultaneously. UW impulses (for explanation of this term see Sect. 1) are strong, but are of short duration, while FLR activity is nearly always present to a certain degree.

2. Even in the absence of distinct UW activity, the latitudinal distribution of the pulsation amplitude may change quickly, within a few minutes or even faster. FLRs are confined to a part of the present array, i.e. they do not cover the whole mid-latitude zone. This fact has already been observed by $\mathrm{Cz}$ Miletits et al. (1990). FLR is more frequently observed at the northern part of the array, at NGK and at WRH.

3. High frequency resolution dynamic spectra detect the local FLRs as activity maxima (spectral peaks) with periods differing from each other by about $10 \%$. Simultaneously, two or more such "lines" may be present at the same station, up to a maximum of five.

4. UW impulses may terminate previously existing oscillations of FLR, and build up new structures. Structures change abruptly rather than by continuous transition.

Even if the very high resolution dynamic spectra produce structures elongated along the time-axis, the observations described by points 3 and 4 show a physical change in the structure of the pulsation spectra, independently of the method used.

\section{May event}

As a second example, the 0800-0900 UT interval, 22 May 1991, is chosen. Figure 12 shows the axes of the

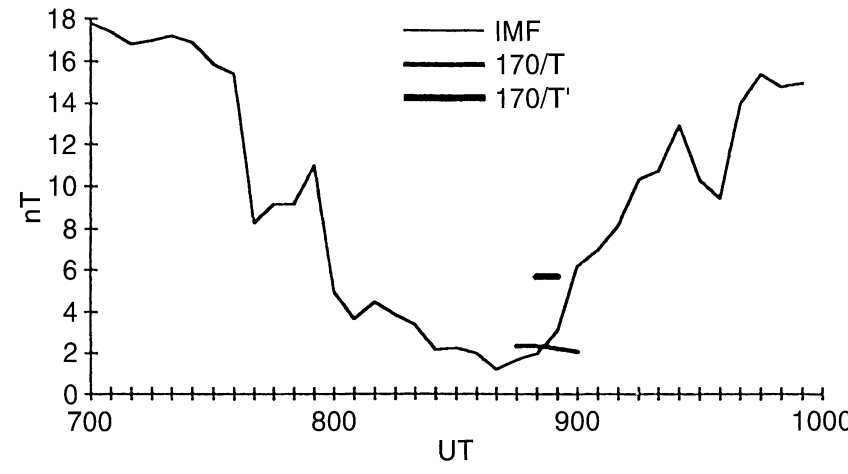

Fig. 13. IMF scalar magnitude during the $3 \mathrm{~h}$ centred around the interval 0800-0900 UT, 22 May, and the observed surface pulsations of supposedly UW origin. In this particular case (expressed as $\left.B^{*}=170 / T\right)$, the long-period ( $T$, about 70-80 s) activity may be of UW origin, the exceptional 30 -s activity $(T)$ with constant period at 0847-0849.5 UT may correspond to a somewhat later IMF value

maximum amplitudes (periods) vs. latitude of the recording stations (WRH data are missing for this interval). In Fig. 13, the latitude-independent periods are plotted vs. time together with the (simultaneous) IMF scalar magnitudes.

The situation in the 22 May event seems to be rather simple: between 0843 and 0846 , there are FLRs along the whole array, at 0847 , i.e. 1 min after the first sample, the situation is different: only UW (with periods of about $30 \mathrm{~s}$ and perhaps with 70-80 s) can be identified. A few minutes later, between 0853 and 0855.5 , the situation has again changed: FLR is present, but the change of the period vs. latitude is somewhat less than normal. Immediately afterwards the latitude dependence of the periods again becomes stronger, not being very different from that during the first short interval (0843).

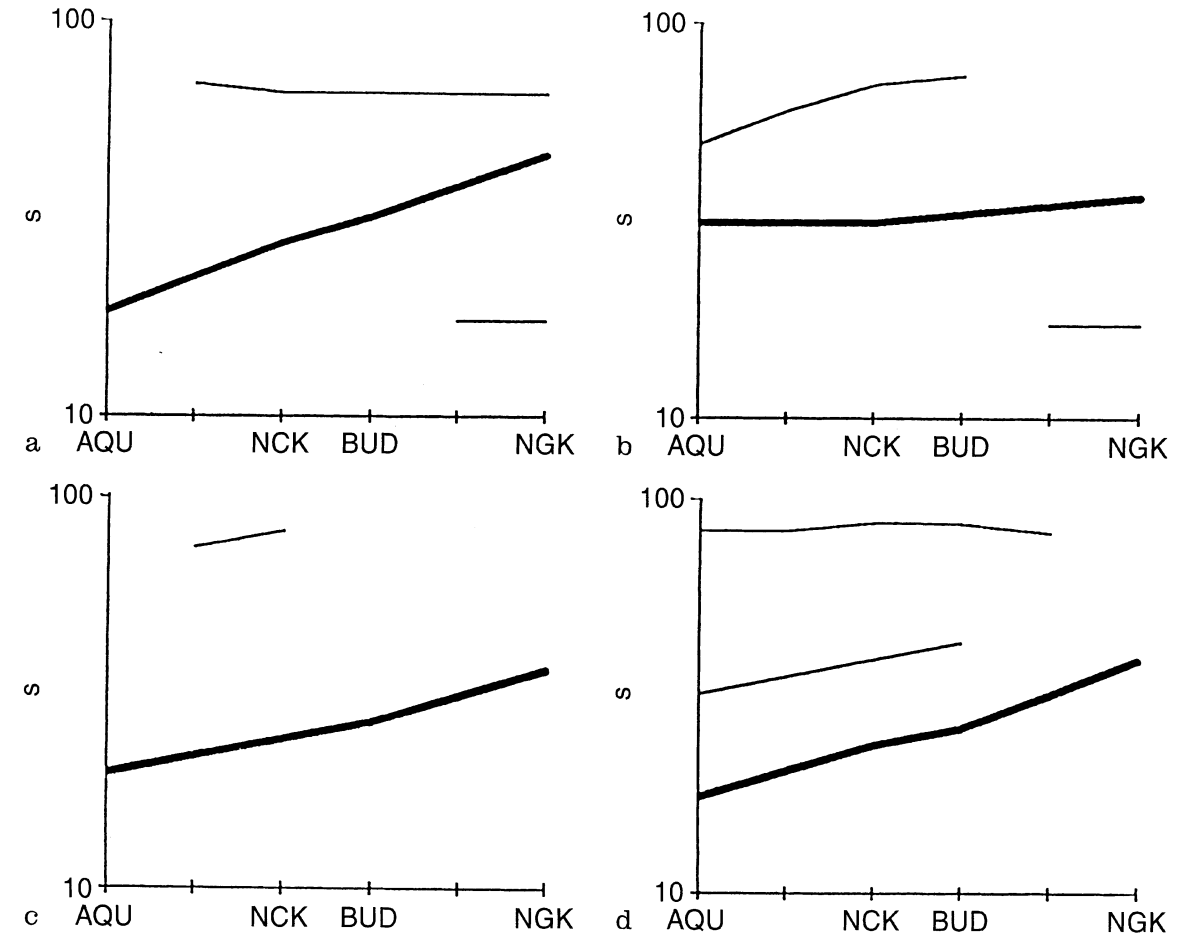

Fig. 12a-d. Location of the amplitude maxima on 22 May 1991, a 0843-0846 UT, b 0847-0849.5 UT, c 0853-0855.5 UT and d 0855.5-0857 UT. The lines indicate the position of amplitude maxima, the thicker the line, the stronger the amplitude maxima. Short periods (about $15 \mathrm{~s}$ ) are only present at NGK, thus in $\mathrm{a}$ and $\mathrm{b}$ the corresponding lines are not necessarily horizontal 
In contrast to this simple picture, however, Fig. 13 shows that the IMF was very weak during this interval, and thus some small $70-80$-s activity present several times during the event (see e.g. in Fig. 12) may be the UW component, while the 30 -s activity appearing around 0848 can only be connected to a later phase of the changing IMF; the shift of about $5 \mathrm{~min}$ is, however, acceptable with reference to the shift experienced during the 18 June event.

Both examples, the 18 June event described in most detail and the 22 May event, have in common that the IMF was very strongly varying during these intervals: moreover, at the end of the interval (22 May) or shortly afterwards (18 June) a shock front reached the vicinity of the Earth.

\section{Other intervals from the 1991 and 1984 arrays}

Thirteen intervals (of a total of 360 recorded) were selected on the basis of the criterion to include different kinds of pulsation activity, with and without period change vs. latitude. This sample includes the 22 May and 18 June, 0800- 0900 UT events, too. According to the 5-min data of the IMF obtained after selection, the intervals with highly variable IMF (difference between maximum and minimum $B$ values more than $3 \mathrm{nT}$ ) proved to contain frequent switches between UW and FLR activity. From the remaining ten events (of less frequent switches between UW- and FLR-type acitivities), only one had similar variability in $B$ (elsewhere the 5 -min values changed within a range less than $1.5 \mathrm{nT}$ ). Even in that exceptional case, there was only one sudden change in the IMF at the middle of the interval without a corresponding change in the period; it is possible that the responsible shock did not affect the Earth.

A survey of a dozen events collected during the functioning of the 1984 array (Cz. Miletits et al., 1990) confirmed that a quick switch between FLR- and UWtype pulsations was then also frequent: the more frequent, the more variable the IMF.

In spite of the complex nature of the pulsation activity during the selected events and without presenting here detailed results, several common characteristics of these events are evident:

1. High variability of the IMF leads to a strong excitation of FLR (2 May, 22 May and 18 June events in 1991).

2. UW and FLR are often mixed, with higher time resolution one will probably find an alternation of both types.

\section{Discussion}

In the present study, the 18 June event served as a basis. In the other twelve events of the 1991 array and in several events of the 1984 array, similar characterics were found. Evidently, the very quick changes between the two types, UW and FLR pulsations, are character- istic for a part of the events, when the variability in the IMF magnitude is also high.

Some of our points go beyond previous work, especially of Waters et al. $(1991,1994)$ and Samson et al. $(1992,1995)$; these papers are used in the following as reference.

1. We suppose that pulsations directly driven by upstream waves are nearly as common as FLR. Otherwise, the connections Pc3-4 period - IMF magnitude and pulsation amplitude - cone angle (e.g. Verõ, 1980) would be hardly interpretable. Moreover, signals having the same period at all latitudes or $L$-values are quite often clearly present, as shown e.g. in Fig. 5. Some connection may also exist between FLR and IMF magnitude (period of UW) due to the fact that FLR can only be excited when the spectrum of the UW contains the local FLR period, and thus a shift in the UW spectrum may shift the location of the zone where FLR is observed, in turn shifting the "global" spectrum of FLR too. This connection is, however, insufficient to explain the experimentally confirmed very close connection between Pc3-4 periods and IMF magnitude, and even less the connection with the cone angle.

2. We found a quick alternation of FLR- and UWtype events, even within intervals which were previously characterized as belonging to any of these two types based on spectra over longer, say hourly intervals. This alternation means, at least in a significant part of the cases studied, that UW impulses come through the magnetosphere and excite FLRs. These FLRs then oscillate with the period of the shell for some time. If there are many UW impulses during an interval, the whole pulsation activity of the interval may take on the character of an UW event, meaning that the actual pulsation period depends on IMF magnitude, and does not change with latitude. Nevertheless, stations at certain latitudes, as e.g. NGK and WRH ( $L$-values $>2.2$ ) in the present array, may exhibit FLRs, even if elsewhere no FLR is observed.

3. The source of the UW pulsations is quite well explained, the excitation of the FLR remains open. It should be considered, however, that the two types react very similarly to changes in the interplanetary medium (Verõ et al., 1995) (at least in a statistical sense, but due to different physical processes); this is why it is very difficult to separate the two types at a single station. Thus, if all pulsations of a longer time-interval are processed statistically, the effects of the solar-wind energy and of IMF parameters on the two types do not differ significantly, as e.g. in the events of the present study (Verõ et al., 1994). Moreover, the response of the two types does not differ significantly from the appearance of UW in the nearEarth space, as shown by Verõ et al. (1994). It seems that both types are connected to processes in the interplanetary medium: changes in the direction of the IMF may lead to changes in the pressure at the magnetopause and cause an UW-impulse. A further argument in favour of a common origin is the impulse-like excitation and termination of FLR structures, discussed in the present paper. The discrete resonant frequencies correspond to particular resonant shells which exist, regardless of the driving 
mechanism, because of a step-wise variation of the plasmasphere density.

4. There are remarkable differences between spectra of longer (e.g. $1 \mathrm{~h}$ ) intervals and instantaneous spectra. In the spectra of long intervals, FLR is often predominant, in spite of possible lower activity (smaller amplitudes), as its period remains the same, while UW has somewhat variable periods. Therefore, the spectral peak corresponding to the UW type becomes diffuse during longer intervals, while it may be dominant in selected short intervals of an UW impulse.

5. There are pulsations with periods inconsistent with these two mechanisms, i.e. both the constant period differs from the period of the expected UW and the latitude-dependent period does not correspond to the actual FLR, first or second harmonic. In some cases, an instantaneous change in the FLR periods (due to a change in the magnetospheric plasma density) may explain these exceptional periods, as e.g. in the 26 April event. In the case of the 18 June event, the periods seem to follow the changes in the IMF, but with a relation different from the normal one (here $237 / B$ instead of $170 / B$ ). According to Le and Russell (1996), such a value of 237 would be consistent with a reasonable large cone angle, i.e. of the order of $45^{\circ}$. Actually, the cone angle was mostly less than $20^{\circ}$ during this interval, with a maximum of $34^{\circ}$. Thus, the unusual factor cannot be explained by this effect. It is to be remarked here that at 0904 UT, i.e. some $4 \mathrm{~min}$ after the end of our 18 June event, very high activity started in the interplanetary medium. The unusual factor may also be connected to the vicinity of the highly disturbed plasma of this event.

The mentioned unusual periods may be due to incorrect data or interpretation (as e.g. a different IMF $B$-value in the immediate vicinity of the Earth from the satellite position), to some shift in the FLR period (due e.g. to exceptional particle densities in the magnetosphere) or also to some additional source of pulsations, possibly flux transfer events or other transient events at the magnetopause.

A comparison of the present results with those obtained by Waters et al. $(1991,1994)$ and Samson et al. $(1992,1995)$ detected the following similar points:

a. A harmonic structure was most often found at the highest-latitude stations both in the Waters et al. study $(L=2.7)$ and in our case (at NGK). A similar structure was absent at lower-latitude stations (Waters et al:: $L=1.8$, here at several stations below 2.2 down to $L=1.5)$.

b. Waters et al. very often found resonant structures at the $L=1.8$ station (on 40 days out of 42 ). In the present case, resonance is practically always present at the higher-latitude stations (NGK and WRH), while at lower-latitudes below $L=1.8$, its occurrence is somewhat less frequent. Nevertheless, the very high occurrence frequency of FLR is in both cases similar.

c. At the lower-latitude station of Waters et al. $(L=1.8)$, rather broadband signals are recorded, with a resonant line at the upper end of the active frequencies. In the present study, the broadband signals are not attributed to magnetospheric noise (as in Waters et al.), but interpreted as signals coming directly from the interplanetary space (UW) without significant change in the spectrum. The different interpretation is based on the fact that this broadband noise has a peak at a frequency statistically related to the magnitude of the IMF. The observation itself is, however, similar.

d. In an earlier work (Verõ and Cz Miletits, 1994) we estimated the surface width of the resonant shells to about $100 \mathrm{~km}$, and this value was also confirmed by the present study based on the period difference between the lines in the high-resolution dynamic spectra; Waters et al. (1991) gave a value of $80 \mathrm{~km}$.

e. Samson et al. $(1992,1995)$ re-analysed the Waters et al. spectra with higher-frequency resolution and found fine spectra which are in accordance with theoretical spectra for the waveguide-driven FLR. These high-resolution spectra are very similar to our dynamic spectra with very high frequency resolution (Fig. 10). This similarity means that there is a similar number of lines (around three) in each case. The width of the spectral window was in the Samson et al. study 1-2 $\mathrm{mHz}$, and in our "normal" dynamic spectra (where the lines do not appear) 5 (at high frequencies) to $2 \mathrm{mHz}$ (at low frequencies). In the dynamic spectra with very high frequency resolution it was $1-0.4 \mathrm{mHz}$, i.e. in the critical range similar to that used by Samson et al.

f. The latitude (or $L$-value) dependence of the structures found in the spectra is very similar in both cases, with an envelope shifted to lower and lower frequencies at higher latitudes, and even certain lines within these broader resonance intervals are identical or similar at neighbouring stations in both cases.

g. In terms of details of the line spectra, the spacing of the lines is in Samson et al. about $4 \mathrm{mHz}$, being roughly $10 \%$ at periods of about $20-25$ s, corresponding to our Fig. 11. Nevertheless, the spacing in frequency remains the same $4 \mathrm{mHz}$ at longer periods, i.e. at 20 $\mathrm{mHz}$, corresponding to a difference of about $20 \%$ at a period of $40 \mathrm{~s}$. This is more than found in our case, but even in the Samson et al. (1995) study the structure is less clear for the first harmonic at higher-latitude stations (ORB in their Fig. 3).

h. A further significant difference is that in our case the line structure is variable, a given configuration remaining stable only for a few minutes, as maximum for a time-interval of some $10 \mathrm{~min}$, while in Samson et al., the structure is given for $30 \mathrm{~min}$. There may be different causes for this difference: either the Samson et al. example is exceptional concerning the length of the stable line structure, or our example is exceptional with the very quick changes of the two types (this is likely, as in the majority of the events such changes are more seldom). In our material, with the different method of analysis, temporal changes are furthermore much emphasized, meaning that peaks found in the power spectra over full hours (Fig. 1) correspond to some "stable" configuration, while "ephemeral" configurations remain observable for short intervals. The latter explanation seems to be more likely, as the spacing of the peaks e.g. in the NGK power spectrum is 
$4.6 \pm 1.0 \mathrm{mHz}$. In Samson et al. (1995. Table 1), the average theoretical spacing for a comparable $L$-value is $4.4 \mathrm{mHz}$, thus the averages correspond quite well. Moreover, in the case of the event 0800-0900 UT, 18 June the IMF was much more variable than normal, and therefore impulses could more often terminate previously existing structures.

i. The frequency/period corresponding to shells of FLRs in the very high resolution dynamic spectra are stable and do not change in time. Transitions between structures occur when an impulse-like increase appears in the pulsation activity.

$\mathrm{k}$. The trapped plasmaspheric mode with turning points at the plasmapause and inside the plasmasphere (plasmatrough mode) has a period of about $15 \mathrm{mHz}$ (Allan et al., 1986). This mode was not found by Samson et al. In the present study, this mode could be present in event 13 from 1984, as well as in two cases, when 60-s activity was found all along the array.

Concerning the missing evidence of the line structure in satellite measurements (Engebretson et al., 1987), the narrow shells found in the present study exclude the possibility of in situ observation. Moreover, the picture seen by a satellite depends on the phase difference between neighbouring shells. Even if all infinitely thin shells are simultaneously excited, the phase difference between the signals of the different shells increases in time; therefore the components of the signal at different $L$-shells become of confused phase situation after a certain time, and a satellite crossing them would not see any regular pulsation. If the shells are of finite thickness and these shells are in phase, then the sinusoidal character remains even for a long time after the excitation. Nevertheless, some indications for rapid changes in Pc3 pulsations have earlier been seen in satellite data (Mier-Jedrzejowicz and Hughes, 1980).

Acknowledgements. The research described in this paper has been supported in Hungary by the Hungarian State Grants OTKA, Nos. 1171 and T19568 and by the Hungarian Space Research Office. The authors thank Dr. Lepping from Goddard Space Flight Center of NASA for the high-resolution IMP-8 data. We also thank Dr. Mursula from the Oulu University in Finland for the 5min interplanetary data, and the referees for valuable comments.

Topical Editor K.-H. Glaßmeier thanks M. Lester and A. Wolfe for their help in evaluating this paper.

\section{Appendix}

The equations for the convolution filter used for the computation of dynamic spectra are:

$$
\begin{aligned}
& F(t)=\frac{1}{2 \pi t}\left(\sin \frac{2 \pi t}{p_{1}}-\sin \frac{2 \pi t}{p_{2}}\right) \cdot\left(\cos \frac{2 \pi t}{T}+1\right), \\
& F(0)=\frac{2 d}{p_{1}}-\frac{2 d}{p_{2}}, \\
& G(t)=\frac{1}{2 \pi t}\left(\cos \frac{2 \pi t}{p_{1}}-\cos \frac{2 \pi t}{p_{2}}\right) \cdot\left(\cos \frac{2 \pi t}{T}+1\right), \\
& G(0)=0,
\end{aligned}
$$

where $t$ is time (of the filter), $F$ is the in-phase, $G$ the outof-phase component of the filtered vector, $d$ is the sampling rate, $p_{1}$ and $p_{2}$ the period limits of the box-car type filter and $T$ the length of truncation (with a Hanning-window). Without going into details of the computation, the time resolution of the filter is controlled by $T$, the frequency/period resolution by $p_{1}$ and $p_{2}$ for a given $d$. Although the two are theoretically independent, in practice they are not. Namely, the length of the filter is most advantageously chosen if the end of the Hanning-window coincides with the first minimum of the filter envelop on the left and right sides of $t=0$. The position of this minimum depends in turn on $p_{1}$ and $p_{2}$; more exactly, on the ratio of the two limits. In the case of "normal" dynamic spectra, they are $10 \%$ less/more than the central period $p$ (in the subsequent filter, the central period also changes by $10 \%$, and thus the whole range of periods is covered by the filter series). Correspondingly, $T=5 p$, and this is the half length of the record from which values are used for the determination of a filtered value.

If we want a higher frequency/period resolution, the distance between $p-p_{1}$ and $p_{2}$ must be less, for example $2 \%$, as in the case of the very high resolution spectra used in the present paper. In this case, however, a higher value must be chosen for $T$ (else the "theoretical" limits $p_{1}$ and $p_{2}$ would be missed too much). An appropriate choice, on the same basis as before, is $T=25 p$.

Thus, if the frequency/period resolution is increased $n$ times, then the time resolution, being proportional to $T$, has to decrease $n$ times too.

\section{References}

Allan, W., E. M. Poulter, and S. P. White, Hydromagnetic wave coupling in the magnetosphere- Plasmapause effects on impulse excited resonances, Planet. Space Sci., 34, 1189-1196, 1986.

Chen L., and A. Hasegawa, A theory of long-period magnetic pulsations, 1. steady-state excitation of field line resonance, $J$. Geophys. Res., 79, 1024-1032, 1974.

Cz. Miletits, J., J. Verõ, J. Szendrõi, P. Ivanova, A. Best, and M. Kivinen, Pulsation periods at mid-latitudes - a seven-station study. Planet. Space Sci., 38, 85-95, 1990.

Engebretson, M. J., L. J. Zanetti, T. A. Potemra, W. Baumjohann, and H. Lühr, Simultaneous observations of Pc3-4 pulsations in the solar wind and in the Earth's magnetosphere, J. Geophys. Res., 92, 10053-10062, 1987.

Gul'elmi, A. U., Diagnostics of the magnetosphere and interplanetary medium by means of pulsations. Space Sci. Rev., 16, 331340,1974

Hughes, W. J., The effect of the atmosphere and ionosphere on long-period magnetospheric micropulsations, Planet. Space Sci., 22, 1157-1172, 1974

Le, G., and Russell, C. T., Solar-wind control of upstream wave frequency, J. Geophys. Res., 101, 2571-2575, 1996.

Mier-Jedrzejowicz, C., and J. Hughes, Phase skipping and packet structure in geomagnetic pulsation signals. J. Geophys. Res., 85, 6888-6892, 1980.

Russell, C. T. and M. M. Hoppe, The dependence of upstream wave periods on the interplanetary magnetic field strength, Geophys. Res. Lett., 8, 615-617, 1981.

Samson, J. C., B. G. Harrold, J. M. Ruohoniemi, R. A. Greenwald, and A. D. M. Walker, Field line resonances associated with MHD waveguides in the magnetosphere, Geophys. Res. Lett., 19, 441-445, 1992. 
Samson, J. C., C. L. Waters, F. W. Menk, and B. J. Fraser, Fine structure in the spectra of low-latitude field line resonances, Geophys. Res. Lett., 22, 2111-2114, 1995.

Southwood, D. J., Some features of field line resonance in the magnetosphere, Planetary Space Sci., 22, 483-491, 1974.

Střeštik, J., Correlations between the parameters of the interplanetary medium and the polarization characteristics of geomagnetic Pc3 and Pi2 pulsations, Trav. Géophys. 35, 55-74, 1987.

Verõ, J., Geomagnetic pulsations and parameters of the interplanetary medium, J. Atm. Terr. Phys., 42, 371-380, 1980.

Verõ, J., Experimental aspects of low-latitude pulsations - a review. J. Geophys., 60, 106-120, 1986.

Verõ, J. and J. Cz. Miletits, Impulsive pulsation events and pulsation beats, J. Atmos. Terr. Phys., 56, 433-445, 1994.

Verõ, J., B. Zieger, and H. Lühr, Upstream waves and surface geomagnetic pulsations. in Solar wind sources of magnetospheric ultra-low-frequency waves, Geophys. Monogr. 81, Am. Geophys. Union, pp. 365-373 1994.

Verõ, J., I. Best, M. Vellante, H. Lühr, M. de Lauretis, L. Hollõ, F. Märcz, and J. Střeštik, Relations of field line resonances and upstream waves and the winter attenuation of pulsations. Ann. Geophysicae, 13, 689-697, 1995

Villante, U., S. Lepidi, M. Vellante, A. J. Lazarus, and R. P. Lepping, Pc3 activity at low geomagnetic latitudes: a comparison with solar wind observations, Planet. Space Sci., 40, 13991408, 1992.

Waters, C. L., F. W. Menk, and B. J. Fraser, The resonance structure of low-latitude Pc3 geomagnetic pulsations, Geophys. Res. Lett., 18, 2293-2296, 1991.

Waters, C. L., F. W. Menk, and B. J. Fraser, Low-latitude geomagnetic field line resonance: experiment and modeling, $J$. Geophys. Res., 99, 17547-17558, 1994.

Yedidia, B. A., M. Vellante, U. Villante, and A. J. Lazarus, A study of the relationship between micropulsations and solar-wind properties, J. Geophys. Res., 96, 3465-3470, 1991.

Yumoto, K., Generation and propagation mechanisms of lowlatitude magnetic pulsations - a review, J. Geophys., 60, 79-105, 1986. 